Check for updates

Cite this: RSC Adv., 2019, 9, 22950

\title{
Ultralight aerogel based on molecular-modified poly $(m$-phenylenediamine) crosslinking with polyvinyl alcohol/graphene oxide for flow adsorption $\dagger$
}

\author{
Yao Chen, ${ }^{\text {ab }}$ Liang Yang, (D) *a Shihao $\mathrm{Xu}^{\text {ab }}$ Shuai Han, ${ }^{\text {ab }}$ Suyun $\mathrm{Chu}^{\text {ab }}$ \\ Zhenyang Wang ${ }^{a}$ and Changlong Jiang (D) *a
}

Poly $(m$-phenylenediamine) is an excellent adsorbent material. Nevertheless, it is difficult to recover and can even generate secondary pollution due to its powder-like form. Aerogels, monolithic three-dimensional structured materials, that are ultralight and have porous properties are efficient at adsorbing contaminants from water and can solve these problems. Here, we synthesized an aerogel based on molecular-modified poly( $m$-phenylenediamine) (mPmPD) crosslinking with polyvinyl alcohol (PVA) and graphene oxide (GO) (GO/mPmPD/PVA). Of note is that 3-aminophenylboronic acid was introduced into the polymer structure to induce a crosslinking reaction between boric acid units and PVA to constrain poly $(m$-phenylenediamine) in the aerogel. The GO/mPmPD/PVA aerogel shows stable mechanical properties in aqueous solution and an effective adsorption capacity for $\mathrm{Ag}(1)$; the maximum $\mathrm{Ag}(\mathrm{l})$ adsorption capacity is $917.41 \mathrm{mg} \mathrm{g}^{-1}$. The mechanism of $\mathrm{Ag}(\mathrm{I})$ adsorption and reduction was clarified in that $A g(I)$ chelated with imine units, and a redox reaction occured between $A g(1)$ and the benzenoid amine units. Furthermore, the GO/mPmPD/PVA aerogel also shows excellent adsorption ability toward methyl orange and Congo red dyes. This GO/mPmPD/PVA aerogel shows enormous potential for application to silver recovery and dye removal due to its low cost, effective adsorption capacity and facile separation with aqueous solution.

Received 4th June 2019

Accepted 14th July 2019

DOI: $10.1039 / c 9 r a 04207 e$

rsc.li/rsc-advances

\section{Introduction}

Silver, a precious metal, has been widely used in photography, coinage, and electroplating, and much wastewater containing $\mathrm{Ag}(\mathrm{I})$ has been discharged as result of these uses. ${ }^{1-4}$ Considering its scarcity, widespread application, and environmental pollution, it is necessary to treat and recover $\mathrm{Ag}(\mathrm{I})$ from wastewater. Compared with other technologies, adsorption is considered to be one of the most appropriate ways to treat metal ions, including $\mathrm{Ag}(\mathrm{I})$, in wastewater. ${ }^{5,6}$ In recent years, many absorbent materials, including clay minerals, ${ }^{7,8}$ activated carbons, ${ }^{9}$ and conducting polymers, ${ }^{5,10-12}$ have been developed. In particular, conducting polymers are considered to be promising materials for treating metal ions due to their strong chelating ability and redox reversibility. ${ }^{12,13}$

One type of conducting polymer, polyphenylenediamines, have aroused a great deal of attention due to their superior

${ }^{a}$ Institute of Intelligent Machines, Chinese Academy of Sciences, Hefei 230031, China. E-mail: yangliang@iim.ac.cn; cljiang@iim.ac.cn

${ }^{b}$ University of Science and Technology of China, Hefei 230026, China

$\dagger$ Electronic supplementary information (ESI) available. See DOI: 10.1039/c9ra04207e conductivity, multifunctionality, high capacity for removal of contaminants and low cost. ${ }^{5,14,15}$ Recently, some reports have suggested that polyphenylenediamines have excellent adsorption ability for $\mathrm{Hg}(\mathrm{I}), \mathrm{Pb}(\mathrm{II}), \mathrm{Cr}(\mathrm{VI}), \mathrm{Ag}(\mathrm{I})$, and dyes., ${ }^{5,1214-16}$ Compared with other polyphenylenediamines, poly ( $m$-phenylenediamine) (PmPD) is considered to be an excellent adsorbent because of its simple synthesis process. The polyreaction of poly (o-phenylenediamine) is only initiated under harsh conditions, where the reaction temperature is above $100{ }^{\circ} \mathrm{C}$, and poly( $p$-phenylenediamine) dissolves in many common solvents, which limits its application in adsorption. However, PmPD has a small specific surface area, which influences the adsorption capacity, and is difficult to separate from water in the practical environment, even causing secondary pollution because of its powdered form. Hence, developing an environmentally friendly, low cost, and efficient method to solve these problems is needed.

Graphene oxide (GO) has attracted much attention in recent years due to its superior specific surface area. ${ }^{17,18}$ Graphene oxide and reduced graphene oxide (rGO) have been used in many fields, such as catalysis, ${ }^{19}$ electrochemical energy storage, ${ }^{20,21}$ and adsorption. ${ }^{5}$ Recently, lots of materials composed of graphene oxide and conducting polymers have 
been synthesized. Poly $(m$-phenylenediamine $) /$ reduced graphene oxide was synthesized by redox reaction for the electrosorption of $\mathrm{Cu}(\mathrm{II})^{22}$ and for adsorbing dyes, ${ }^{5}$ and polypyrrole/ graphene oxide ${ }^{23}$ was prepared to remove $\mathrm{Cr}(\mathrm{vI})$ from aqueous solution. Furthermore, three-dimensional (3D) structures of graphene oxide and reduced graphene oxide are considered to have more significance in practical applications. Recently, some reports have suggested that aerogels self-assembled by polyaniline and graphene oxide nanosheets possess significant performances in electrochemical energy storage and catalysis. ${ }^{21,24,25}$ It is noteworthy that the advantages of ultralight weight, high porosity and high specific surface area are also of benefit for adsorption. Therefore, the aerogel is considered to be one of the most promising materials for recovery of metal ions from wastewater and to solve the water pollution problem. However, reduced graphene oxide-based aerogels are not hydrophilic and do not have good enough mechanical properties for application in aqueous solution.

Here, we synthesized an aerogel based on molecularmodified poly $(m$-phenylenediamine) (mPmPD) crosslinking with polyvinyl alcohol (PVA) and graphene oxide (GO). PVA, a flexible and tough polymer material, has already been used in many fields; for instance, in pharmaceuticals and as a flexible capacitor. ${ }^{26,27}$ Therefore, we chose PVA to strengthen the mechanical properties and hydrophilicity of the aerogel, a GO sheet to be the skeleton frame, and MPMPD to be the functional section. Inspired by the crosslinking reaction between boric acid and PVA, we introduced 3-aminophenylboronic acid (ABA) into the polymerization to modify PmPD at a molecular level to induce crosslinking with PVA. ${ }^{28}$ A typical B 1 s peak appears in the wide X-ray photoelectron spectrum of the GO/mPmPD/PVA aerogel, suggesting that boric acid groups exist in the aerogel. The GO/mPmPD/PVA aerogel shows stable mechanical properties in aqueous solution and high adsorption capacity for $\mathrm{Ag}(\mathrm{I})$. The maximum Ag(I) adsorption capacity is $917.41 \mathrm{mg} \mathrm{g}^{-1}$. The mechanism of $\mathrm{Ag}(\mathrm{I})$ adsorption and reduction is explained by chelation between $\mathrm{Ag}(\mathrm{I})$ and imine units and the redox reaction between $\mathrm{Ag}(\mathrm{I})$ and benzenoid amine units. Furthermore, the $\mathrm{GO} / \mathrm{mPmPD} / \mathrm{PVA}$ aerogel also shows excellent adsorption ability for methyl orange and Congo red dyes. In a word, this GO/ mPmPD/PVA supramolecular-structure aerogel shows enormous potential in applications for silver recovery and dye removal due to its low cost, high adsorption capacity and facile separation with aqueous solution.

\section{Experimental}

\subsection{Materials}

Ammonium persulfate (APS), polyvinyl alcohol (PVA) (MW 1750), graphite powder, hydrogen peroxide $\left(\mathrm{H}_{2} \mathrm{O}_{2}\right)$, potassium permanganate $\left(\mathrm{KMnO}_{4}\right)$, sulfuric acid $\left(\mathrm{H}_{2} \mathrm{SO}_{4}\right)$, phosphoric acid $\left(\mathrm{H}_{3} \mathrm{PO}_{4}\right)$, and silver nitrate $\left(\mathrm{AgNO}_{3}\right)$ were analytical pure grade and purchased from Shanghai Sinopharm Chemical Reagent Corporation. Methyl orange, Congo red, $m$-phenylenediamine (mPD), and 3-aminophenylboronic acid (ABA) were purchased from Aladdin Industrial Corporation and were analytical pure grade. Ultrapure water $(18.2 \mathrm{M} \Omega \mathrm{cm})$ was used to prepare aqueous solutions for all experiments.

\subsection{Preparation of GO/mPmPD/PVA supramolecular- structure aerogel}

The GO/mPmPD/PVA supramolecular-structure aerogel was prepared through a one-step, in situ synthesis process. GO was prepared according to the improved Hummers' method, ${ }^{29}$ and dispersed in ultrapure water and shaken ultrasonically for 3 hours to obtain a $2.5 \mathrm{mg} \mathrm{mL}^{-1}$ uniform GO suspension. A certain amount of $\mathrm{mPD}$ and ABA were added to the GO suspension and vigorously stirred for 2 hours at $25{ }^{\circ} \mathrm{C}$. Then, a certain amount of PVA solution ( $\left.50 \mathrm{mg} \mathrm{mL}^{-1}\right)$ was added into the aqueous solution and stirred for 1 hour at $25{ }^{\circ} \mathrm{C}$. After that, oxidant solution with APS (oxidant: monomer, $\sim 1: 1$ ) was added to the system drop by drop to initiate the polymerization reaction, and the reaction solution was steadily stirred for 4 hours at $25{ }^{\circ} \mathrm{C}$. The PmPD and GO sheets would be attracted together via strong $\pi-\pi$ stacking interactions. The mix suspension was transferred to an oven for crosslinking for 2 hours at $65{ }^{\circ} \mathrm{C}$. The mechanism of polymerization and crosslinking is shown in Scheme 1, where the boric acid units in PmPD can induce crosslinking between PmPD and PVA at the molecular scale. After the polymerization reaction and crosslinking, GO/mPmPD/PVA hydrogel samples were formed by freeze-thaw. To investigate the effect of ABA in the process,
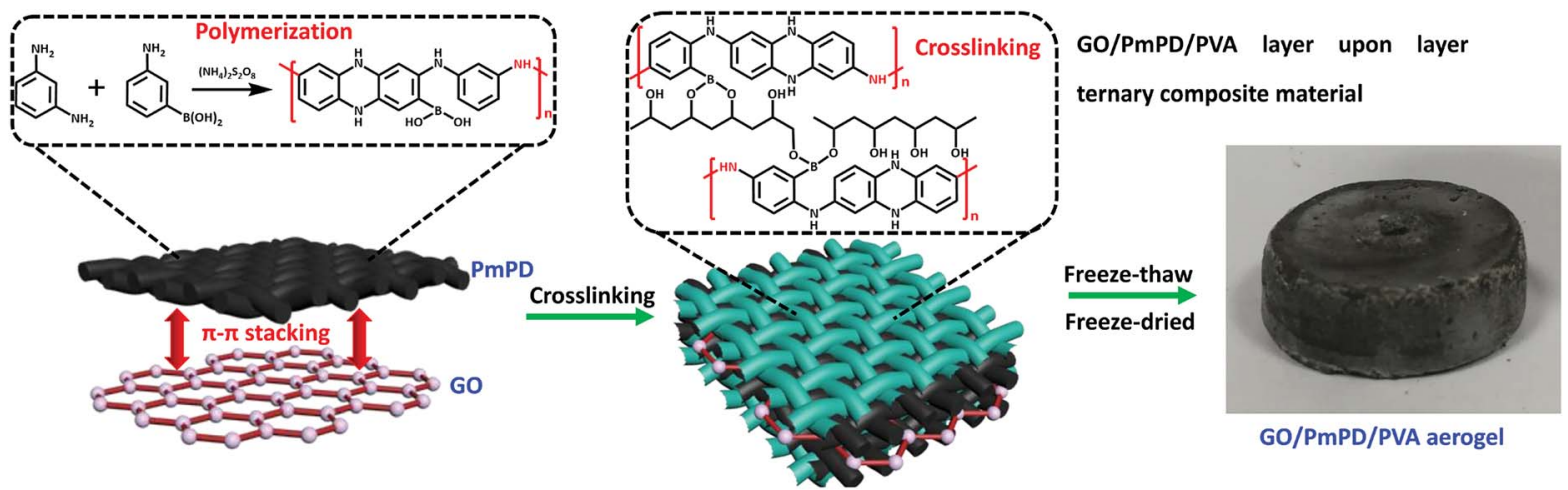

Scheme 1 Schematic diagram of the synthesis mechanism of the GO/mPmPD/PVA aerogel. 
system reactions with different $\mathrm{ABA}$ content were carried out. Photographs of samples after freeze-thaw are shown in Fig. $\mathrm{S} 1, \uparrow$ and the optimal ratio of $\mathrm{ABA}: \mathrm{mPD}$ is $1: 9 . \mathrm{GO} /$ $\mathrm{mPmPD} / \mathrm{PVA}$ hydrogel samples were washed with plenty of ultrapure water to remove impurities and then freeze-dried for 48 hours to obtain the final GO/mPmPD/PVA supramolecularstructure aerogels. Aerogels of various GO : mPD/ABA : PVA ratios were synthesized to find the most appropriate ratio. It is noteworthy that the sample cannot form an aerogel when the ratio between $\mathrm{mPD} / \mathrm{ABA}$ and PVA exceeds $4: 6$. Considering PmPD as the main functional polymer, the aerogel of ratio GO : mPD/ABA : PVA $=1: 4: 6$ was used in the following experiments.

\subsection{Adsorption experiment}

A series of $\mathrm{Ag}(\mathrm{I})$ adsorption experiments were designed. In brief, $25 \mathrm{mg} \mathrm{GO} / \mathrm{mPmPD} / \mathrm{PVA}$ aerogel was immersed in $20 \mathrm{~mL} \mathrm{Ag}(\mathrm{I})$ solutions with a series of different concentrations. Tweezers were used to take out the GO/mPmPD/PVA aerogel after the system had been shaken for enough time at a constant temperature. The effect of initial $\mathrm{pH}$, temperature, and adsorption time were also investigated. The adsorbed amount was calculated via the following formula:

$$
q_{\mathrm{e}}=\frac{\left(C_{0}-C_{\mathrm{e}}\right) V}{m}
$$

where $q_{\mathrm{e}}\left(\mathrm{mg} \mathrm{g}^{-1}\right)$ is the amount of adsorbed $\mathrm{Ag}(\mathrm{I})$ at equilibrium, $C_{0}$ and $C_{\mathrm{e}}\left(\mathrm{mg} \mathrm{L}^{-1}\right)$ are the concentrations of the $\operatorname{Ag}(\mathrm{I})$ solution at the start time and equilibrium time, $V(\mathrm{~mL})$ is the volume of the solution and $m(\mathrm{mg})$ is the dry mass of the adsorbent. The concentration of $\mathrm{Ag}(\mathrm{I})$ was determined by an inductively coupled plasma emission spectrometer.

$\mathrm{GO} / \mathrm{mPmPD} / \mathrm{PVA}$ aerogel was also used to remove methyl orange and Congo red dyes, and the adsorption properties for methyl orange and Congo red were investigated. Briefly, $10 \mathrm{mg}$ $\mathrm{GO} / \mathrm{mPmPD} / \mathrm{PVA}$ aerogel was put into $20 \mathrm{~mL}$ methyl orange solutions with a series of different concentrations. The concentrations of methyl orange in the solutions were determined using a UV-vis spectrophotometer at $464 \mathrm{~nm}$ after shaking for 36 hours. The adsorption properties for Congo red
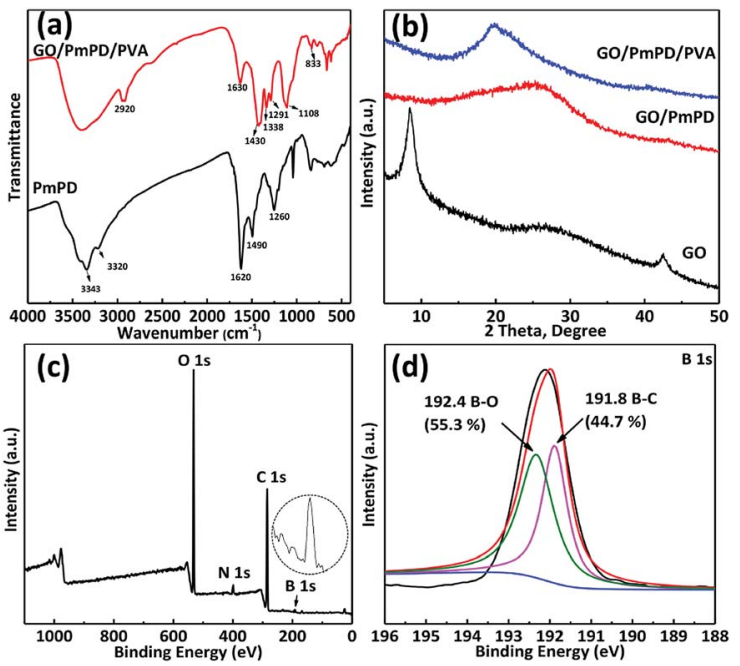

Fig. 2 (a) FT-IR spectra of PmPD and the GO/PmPD/PVA aerogel. (b) XRD patterns of GO, GO/PmPD and the GO/PmPD/PVA aerogel. (c) Wide XPS spectrum of the GO/PmPD/PVA aerogel. (d) High-resolution spectrum of $B$ 1s.

were determined using the same method as for methyl orange, with the concentrations determined at $497 \mathrm{~nm}$.

\subsection{Characterization}

The morphology of the GO/mPmPD/PVA aerogel was obtained by scanning electron microscopy (SEM, Sirion 200, FEI Co., USA). The concentration of Ag(I) was determined by inductively coupled plasma emission spectrometry (ICP-ES, Optima 7300 DV, PerkinElmer Inc., Electron Co., USA). The concentration of methyl orange and Congo red were determined using a UV-vis spectrophotometer (UV-2550, Shimadzu Co., Japan). The X-ray diffraction patterns of GO, GO/PmPD, and GO/mPmPD/PVA aerogel were determined using $\mathrm{CuK} \alpha$ radiation and an X-ray diffractometer (XRD, X'Pert PRO, PANalytical B.V., The Netherlands). Fourier-transform-infrared (FT-IR) spectra were measured using an infrared spectrophotometer (FT-IR, Nicolet 8700, Thermo Scientific Instrument Co., USA). X-ray photoelectron spectra were recorded using an X-ray photoelectron
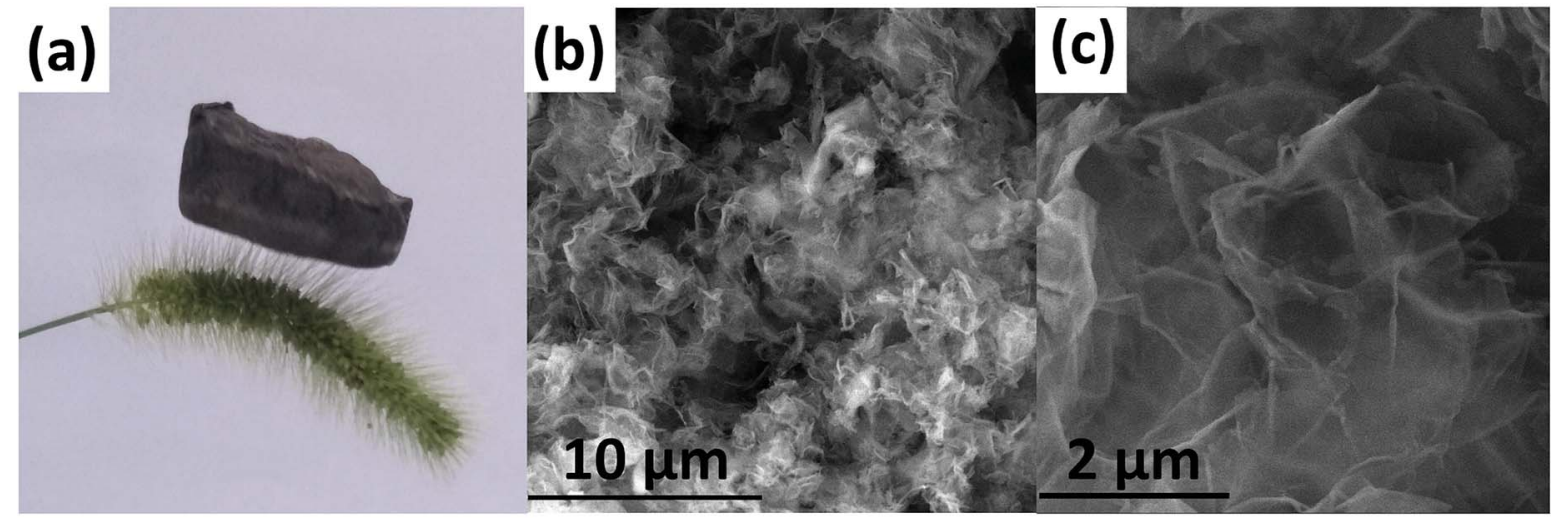

Fig. 1 (a) Photograph of GO/PmPD/PVA aerogel, (b) and (c) SEM images of the GO/PmPD/PVA aerogel. 


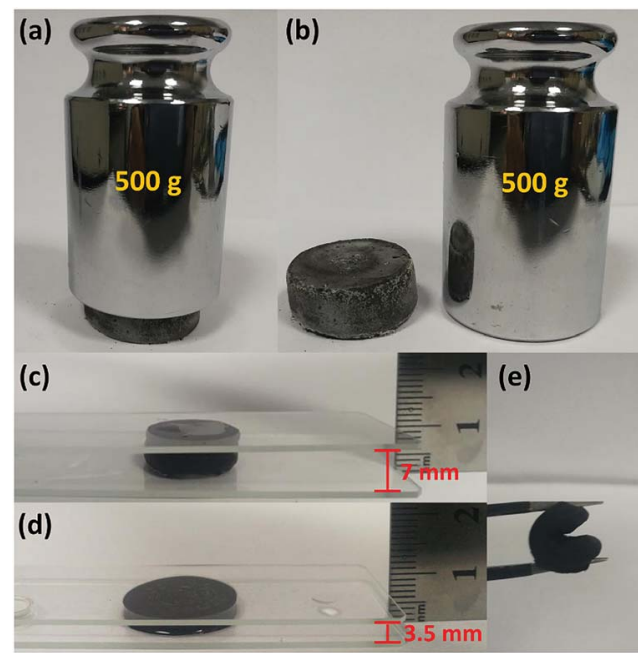

Fig. 3 ( $a$ and b) Photographs of the strong GO/PmPD/PVA aerogel with supporting weight. (c-e) Photographs of the GO/PmPD/PVA aerogel with stable mechanical properties after shaking for 36 hours in aqueous solution.

spectrometer (XPS, ESCALAB 250, Thermo-VG Scientific Co., USA). The pore size, pore volume and surface area were measured by accelerated surface area and porosimetry (Tristar II 3020M, Micromeritics Instrument Co., USA). Thermogravimetric analysis (TGA) was carried out using a thermoanalyser at a heating rate of $10{ }^{\circ} \mathrm{C} \mathrm{min}^{-1}$ under an air atmosphere flow (TGA Q5000IR, TA Instrument Co., USA).

\section{Results and discussion}

\subsection{Characterization of GO/mPmPD/PVA aerogel}

As shown in Fig. 1(a), the prepared ultralight GO/mPmPD/PVA aerogel can be supported on the slim fibers of Setaria viridis, and the fibers do not even deform under the pressure. SEM images of the aerogel with a ratio of $1: 4: 6$ between GO, MPD/ ABA, and PVA are presented in Fig. 1(b) and (c). The morphologies of other samples with various ratios of GO, mPD/ABA, and
PVA are shown in Fig. S2. $\dagger$ In the SEM images in Fig. 1(b) and (c), a lamellar structure is evident with many spaces in the GO/ $\mathrm{mPmPD} / \mathrm{PVA}$ aerogel. This may be attributed to the GO skeleton and crosslinking between PVA and PmPD. The pore size, pore volume, and surface area of PmPD and GO/mPmPD/PVA aerogels are given in Table S1. $\dagger$ The surface area of the GO/mPmPD/ PVA aerogel is $23.49 \mathrm{~m}^{2} \mathrm{~g}^{-1}$, which is much higher than the surface area of PmPD $\left(8.36 \mathrm{~m}^{2} \mathrm{~g}^{-1}\right)$. This higher surface area is beneficial for adsorption. ${ }^{30}$

The FT-IR spectra of PmPD and the GO/mPmPD/PVA aerogel are shown in Fig. 2(a). For comparison, the spectra of GO sheets, PVA and GO/PmPD are presented in Fig. S3. $\dagger$ As shown in the spectrum of PmPD, the broad bands at $3343 \mathrm{~cm}^{-1}$ and $3220 \mathrm{~cm}^{-1}$ correspond to the $-\mathrm{NH}-$ stretching vibration. The sharp peak at $1620 \mathrm{~cm}^{-1}$ can be associated with the quinoid imine and the peak at $1490 \mathrm{~cm}^{-1}$ can be associated with the benzenoid amine. The peak at $1257 \mathrm{~cm}^{-1}$ can be assigned to the stretching vibration of $\mathrm{C}-\mathrm{N}$ in benzenoid amine groups. ${ }^{31}$ Quinoid imine and benzenoid amine groups are the most important functional groups for adsorption. In the spectrum of the GO/mPmPD/PVA aerogel, the peak at $1430 \mathrm{~cm}^{-1}$ can be associated with the amine of PmPD. The peak at $1291 \mathrm{~cm}^{-1}$ can be assigned to $\mathrm{B}-\mathrm{O} .^{32}$ The peak around $2920 \mathrm{~cm}^{-1}$ can be assigned to the $\mathrm{C}-\mathrm{H}$ of $\mathrm{PVA}^{26}$ The peak at $1108 \mathrm{~cm}^{-1}$ can be assigned to the $\mathrm{C}-\mathrm{O}$ of GO and PVA, and the peak at $1630 \mathrm{~cm}^{-1}$ is associated with the $\mathrm{C}=\mathrm{O}$ of $\mathrm{GO}$ and the quinoid imine of PmPD. ${ }^{26,31,33}$

The XRD patterns of GO, GO/PmPD and GO/mPmPD/PVA aerogel are shown in Fig. 2(b). In the pattern for GO, the typical diffraction peak at about $10^{\circ}$ can be assigned to the interlayer space in $\mathrm{GO}^{34}$ In the pattern for GO/PmPD, this diffraction peak at $10^{\circ}$ disappeared and a new broad diffraction peak appeared at about $25^{\circ}$, suggesting the amorphous nature of PmPD. ${ }^{5}$ Compared with GO/PmPD, the broad diffraction peak in the GO/mPmPD/PVA aerogel shifted to about $20^{\circ}$, after crosslinking between boric acid groups and PVA. As noted in some previous reports, ${ }^{\mathbf{1 4}, 31}$ a shift in the diffraction peak of polymers to a low angle signifies an increase in their amorphous nature. Due to the disordered and loose-knit piles of the
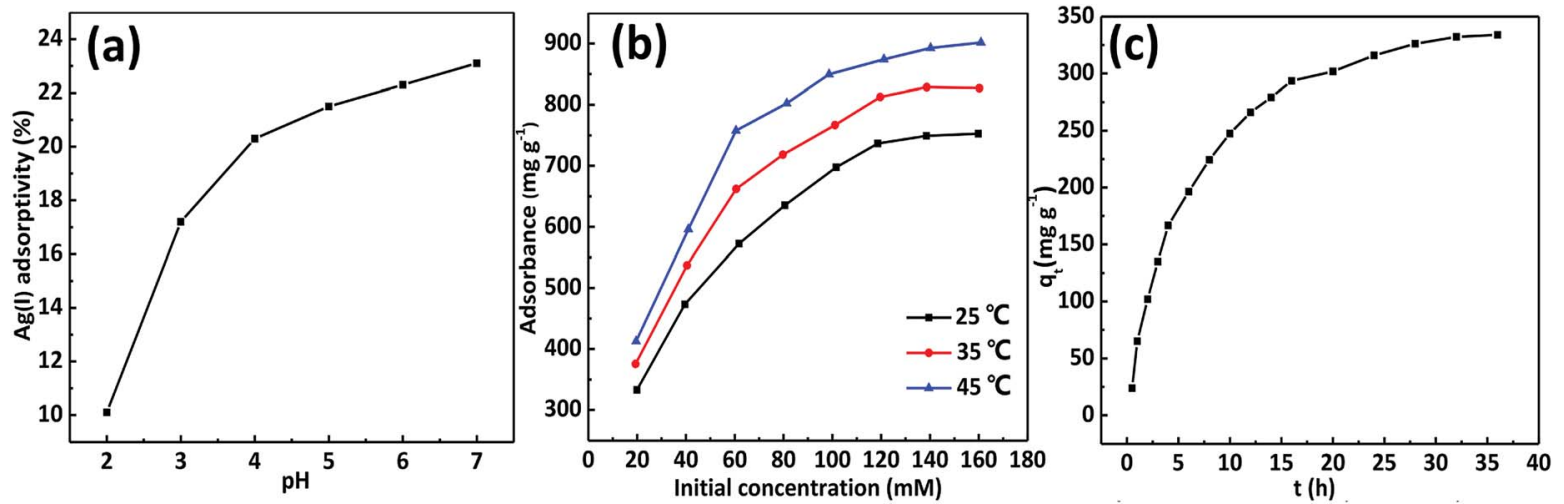

Fig. 4 (a) Effect of $\mathrm{pH}$ on $\mathrm{Ag}\left(\right.$ (I) adsorption by the GO/PmPD/PVA aerogel (temperature, $25^{\circ} \mathrm{C}$; initial concentration, $20 \mathrm{mM}$; dose, $25 \mathrm{mg}$; volume, $20 \mathrm{~mL}$; adsorption time, $36 \mathrm{~h}$ ). (b) Effects of initial concentration and temperature on $\mathrm{Ag}(\mathrm{I})$ adsorption (dose, $25 \mathrm{mg}$; volume, $20 \mathrm{~mL} \mathrm{pH}, 7.0$; adsorption time, $36 \mathrm{~h}$ ). (c) Effect of contact time on $\mathrm{Ag}\left(\right.$ (I) adsorption (dose, $25 \mathrm{mg}$; volume, $20 \mathrm{~mL} ; \mathrm{pH}, 7.0$, temperature, $25^{\circ} \mathrm{C}$; concentration, 20 $\mathrm{mM})$. 
Table 1 Isotherm model equations for Ag(I) adsorption

\begin{tabular}{lllllll}
\hline & \multicolumn{2}{l}{ Langmuir model } & & & \multicolumn{2}{c}{ Freundlich model } \\
\cline { 2 - 5 } Temperature, $T\left({ }^{\circ} \mathrm{C}\right)$ & $q_{\max }\left(\mathrm{mg} \times \mathrm{g}^{-1}\right)$ & $K\left(\mathrm{~L} \times \mathrm{mg}^{-1}\right)$ & $R^{2}$ & & $K_{\mathrm{f}}$ & $n$ \\
\hline 25 & 917.413 & 0.00031 & 0.997 & 21.657 & 2.678
\end{tabular}

supramolecular chains, this amorphous structure is suitable for permeation and adsorption of ions onto the GO/mPmPD/PVA aerogel.

In order to further confirm the existence of boric acid groups, XPS was used to characterize the GO/mPmPD/PVA aerogel. As shown in the XPS results (Fig. 2(c)), the typical B 1s peak appearing in the wide spectrum of the GO/mPmPD/PVA aerogel means that ABA has successfully participated in the reaction system. In the high-resolution $B$ 1s spectrum (Fig. 2(d)), the peak at $191.8 \mathrm{eV}$ corresponds to B-C and the peak at $192.4 \mathrm{eV}$ can be assigned to $\mathrm{B}-\mathrm{O} .^{32}$ This suggests that the boric acid group plays a bridge-like role between PmPD and PVA. The TGA curve of for the GO/mPmPD/PVA aerogel is shown in Fig. $\mathrm{S} 4, \dagger$ and the result suggests that the GO/mPmPD/PVA aerogel has good thermostability for practical applications.

As shown in Fig. 3(a) and (b), the GO/mPmPD/PVA aerogel can support a weight of $500 \mathrm{~g}$, which is about 2500 times its own weight. It is obvious that $\mathrm{GO} / \mathrm{mPmPD} / \mathrm{PVA}$ aerogel has strong mechanical properties. Fig. 3(c)-(e) show the stable mechanical properties after shaking at $300 \mathrm{rpm}$ for 36 hours in aqueous solution. In Fig. 3(c) and (d), the GO/mPmPD/PVA aerogel is still intact and a lot of water was squeezed out after hard pressing. We found that the GO/mPmPD/PVA aerogel can bend and fold without any breakages (see Fig. 3(e)), which suggests that the aerogel is very tough. In a word, GO/mPmPD/PVA aerogel has excellent mechanical properties and hydrophilicity for practical applications in aqueous solution.

\subsection{Properties of $\mathbf{A g}(\mathrm{I})$ adsorption}

As presented in Fig. 4(a), the pH was controlled from 2.0 to 7.0 by $\mathrm{HCl}$ to investigate the effect of $\mathrm{pH}$ for $\mathrm{Ag}(\mathrm{I})$ adsorption by the $\mathrm{GO} / \mathrm{mPmPD} / \mathrm{PVA}$ aerogel. Obviously, the adsorptivity of $\mathrm{Ag}$ (I) in a strong acid environment is much lower than in a neutral environment. More specifically, the adsorptivity of $\mathrm{Ag}(\mathrm{I})$ decreases rapidly, from $20.3 \%$ to $10.1 \%$, when the $\mathrm{pH}$ is reduced from 4.0 to 2.0 . On the other hand, the adsorptivity of $\mathrm{Ag}(\mathrm{I})$ only increases by $2.8 \%$ with a rise in $\mathrm{pH}$ from 4.0 to 7.0 . This means that a weak acid or neutral environment is more suitable for adsorbing $\mathrm{Ag}(\mathrm{I})$ using the GO/mPmPD/PVA aerogel.

The effect of the initial Ag(I) concentration and temperature for adsorption of Ag(I) are shown in Fig. 4(b). As the initial Ag(I) concentration increases, the adsorption capacity of $\operatorname{Ag}(\mathrm{I})$ increases, as a result of the increase in contact between $\mathrm{Ag}(\mathrm{I})$ and active sites in the GO/mPmPD/PVA aerogel. The adsorption capacity tends toward a stabilized value when the initial $\mathrm{Ag}(\mathrm{I})$ concentration is higher than $120 \mathrm{mM}$. Meanwhile, the adsorption capacity of $\mathrm{Ag}(\mathrm{I})$ rises from $752.67 \mathrm{mg} \mathrm{g}^{-1}$ to $901.44 \mathrm{mg} \mathrm{g}^{-1}$ as the temperature rises from $25{ }^{\circ} \mathrm{C}$ to $45{ }^{\circ} \mathrm{C}$, indicating that high temperature is beneficial for $\mathrm{Ag}(\mathrm{I})$ adsorption. In order to achieve the maximum adsorption capacity of $\mathrm{Ag}(\mathrm{I})$ at room temperature and to figure out how the adsorbates interact with the adsorbents, a Langmuir model and a Freundlich model were used to analyze the adsorption isotherm, and these are shown in Fig. S5. $\uparrow$ The relative parameters of the models are listed in Table 1. The experimental data fit better with the Langmuir model through comparing the correlation coefficients $\left(R^{2}\right), 0.997$ for the Langmuir model and 0.976 for the Freundlich model, which indicates adsorption of $\mathrm{Ag}(\mathrm{I})$ in a monolayer. To prove the effective adsorption capacity GO/ mPmPD/PVA aerogel for adsorption of $\mathrm{Ag}(\mathrm{I})$, a comparison between GO/mPmPD/PVA aerogel and other adsorbents is shown in Table S2. $\dagger$

The effect of contact time for $\mathrm{Ag}(\mathrm{I})$ adsorption is shown in Fig. 4(c). The adsorption capacity of $\mathrm{Ag}(\mathrm{I})$ increases rapidly in the initial 15 hours, then gradually slows down and tends to become stable. Moreover, the behavior of $\mathrm{Ag}(\mathrm{I})$ adsorption on the GO/mPmPD/PVA aerogel was analyzed using two kinetic equations and is presented in Fig. S6. $\uparrow$ The fitting results for the two equations are shown in Table 2 . The correlation coefficient of the pseudo-second-order kinetic equation (0.996) is higher than the correlation coefficient of the pseudo-first-order equation (0.960), suggesting that the Ag(I) ions are adsorbed mainly by chemisorption.

\subsection{Adsorption mechanism of $\operatorname{Ag}(\mathrm{I})$ with the GO/mPmPD/ PVA aerogel}

In order to clarify the adsorption mechanism of the Ag(I), XPS was used to characterize the GO/mPmPD/PVA aerogel before and after adsorption of $\mathrm{Ag}(\mathrm{I})$, and the spectra are presented in Fig. 5. After adsorption, an $\mathrm{Ag} 3 \mathrm{~d}$ peak appeared in the wide spectrum verifying that $\mathrm{Ag}$ has been adsorbed onto the $\mathrm{GO} /$ mPmPD/PVA aerogel. Furthermore, we found two peaks for Ag

Table 2 Summary of kinetic parameters for $\mathrm{Ag}(1)$ adsorption

\begin{tabular}{llllllll}
\hline & \multicolumn{2}{l}{ Pseudo-first-order } & & \multicolumn{2}{l}{ Pseudo-second-order } \\
\cline { 2 - 3 } Temperature, $T\left({ }^{\circ} \mathrm{C}\right)$ & $q_{\mathrm{e}}\left(\mathrm{mg} \times \mathrm{g}^{-1}\right)$ & $K_{1}\left(\mathrm{~g} \times \mathrm{mg}^{-1} \times \mathrm{L}^{-1}\right)$ & $R^{2}$ & & $q_{\mathrm{e}}\left(\mathrm{mg} \times \mathrm{g}^{-1}\right)$ & $K_{1}\left(\mathrm{~g} \times \mathrm{mg}^{-1} \times \mathrm{L}^{-1}\right)$ & $R^{2}$ \\
\hline 25 & 336.127 & 0.00231 & 0.960 & 395.257 & $6.4 \times 10^{-6}$
\end{tabular}



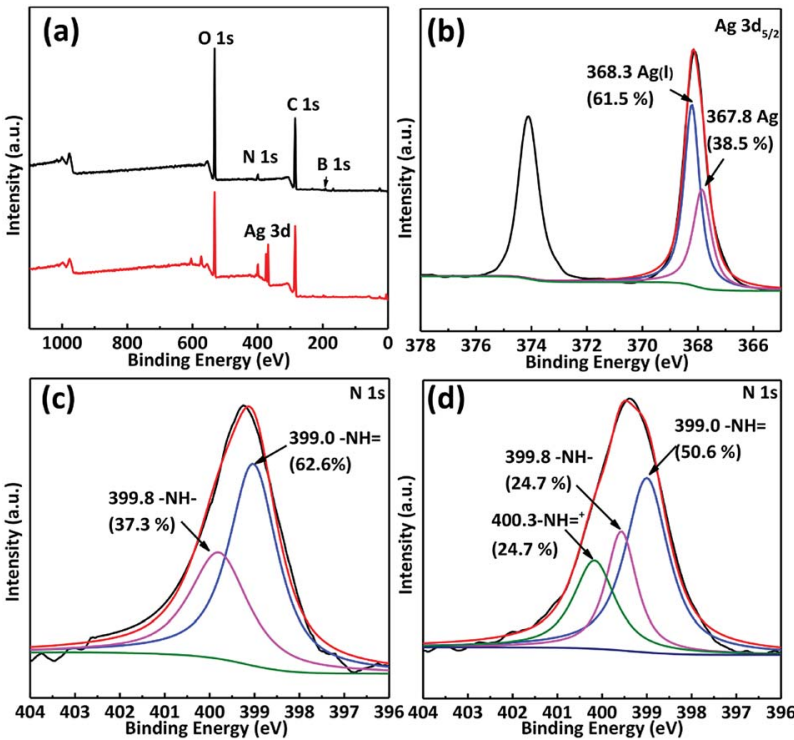

Fig. 5 (a) Wide XPS spectra of the GO/mPmPD/PVA aerogel before and after adsorption of $\mathrm{Ag}(\mathrm{I})$. (b) $\mathrm{Ag} 3 \mathrm{~d}_{5 / 2}$ high-resolution spectrum. (c) $\mathrm{N} 1 \mathrm{~s}$ high-resolution spectrum before adsorption of $\mathrm{Ag}(\mathrm{I})$. (d) $\mathrm{N} 1 \mathrm{~s}$ high-resolution spectrum after adsorption of $\mathrm{Ag}(\mathrm{I})$.

at $367.8 \mathrm{eV}$ and $368.3 \mathrm{eV}$ in the high-resolution $\mathrm{Ag} 3 \mathrm{~d}_{5 / 2}$ spectrum, which are assigned to $\mathrm{Ag}$ (38.5\%) and $\mathrm{Ag}(\mathrm{I})(61.5 \%)$, respectively. This means that a portion of the $\mathrm{Ag}(\mathrm{I})$ ions has been reduced to $\mathrm{Ag}$ by the GO/mPmPD/PVA aerogel. Correspondingly, the proportion of benzenoid amine (-NH-) at $399.8 \mathrm{eV}$ decreased from $37.3 \%$ to $24.7 \%$ after adsorption of $\mathrm{Ag}(\mathrm{I})$, as shown in Fig. 5(c) and (d). Simultaneously, a new peak $\left(-\mathrm{NH}={ }^{+}\right)$ emerged at $400.3 \mathrm{eV}$, which can be attributed to chelation between the quinoid imine $(-\mathrm{NH}=)$ and $\operatorname{Ag}(\mathrm{I}) \cdot{ }^{31}$ Therefore, the mechanism of $\operatorname{Ag}(\mathrm{I})$ adsorption may be explained by the following three features. (1) Part of the $\mathrm{Ag}$ (I) is reduced to $\mathrm{Ag}$ and part of the benzenoid amine $(-\mathrm{NH}-)$ is transformed to the quinoid imine $(-\mathrm{NH}=)$ during a redox reaction. (2) A number of $\mathrm{Ag}(\mathrm{I})$ ions chelate with newly emerging and pre-existing quinoid imine $(-\mathrm{NH}=)$ to form $-\mathrm{NH}={ }^{+} .{ }^{31}$ (3) A small amount of the $\mathrm{Ag}(\mathrm{I})$ ions are adsorbed onto the GO/mPmPD/PVA aerogel through physical adsorption and through oxygen-containing functional groups such as hydroxyl and carboxyl. ${ }^{26,33}$ The redox reaction and chelation are shown in Scheme 2.

\subsection{Properties of dye adsorption}

The adsorption properties of GO/mPmPD/PVA aerogel with respect to dyes are shown in Fig. 6. The concentrations of

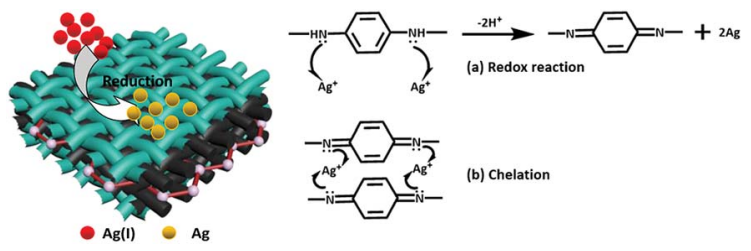

Scheme 2 Schematic illustration of the redox reaction and chelation during $\mathrm{Ag}(\mathrm{I})$ adsorption.
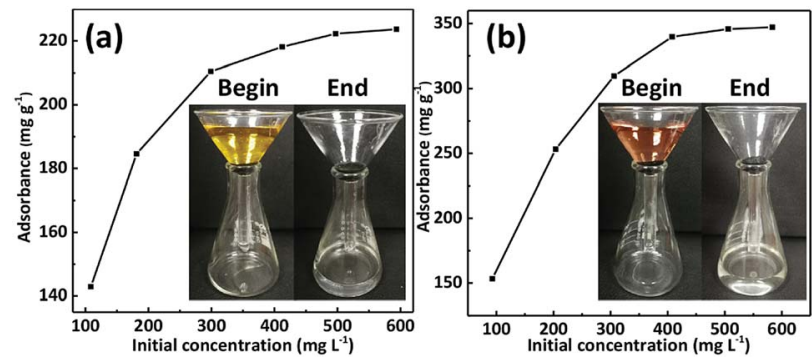

Fig. 6 Adsorption properties of the GO/PmPD/PVA aerogel for (a) methyl orange and (b) Congo red (temperature, $25^{\circ} \mathrm{C}$; dose, $10 \mathrm{mg}$; volume, $20 \mathrm{~mL} ; \mathrm{pH}, 7.0$; adsorption time, 36 h). Insets: methyl orange solution and Congo red solution changed into clean water after flowing through the GO/PmPD/PVA aerogel $(\mathrm{pH}, 7.0$; initial concentration, $10 \mathrm{mg} \mathrm{L}^{-1}$ ).

methyl orange solution and Congo red solution were determined using a UV-vis spectrophotometer at $464 \mathrm{~nm}$ and $497 \mathrm{~nm}$, respectively. The adsorbed amounts for both methyl orange and Congo red increase rapidly with increase in the initial concentration, when the initial concentrations are below $300 \mathrm{mg} \mathrm{L}^{-1}$. When the initial concentrations exceed $300 \mathrm{mg} \mathrm{L}^{-1}$, the amounts adsorbed increase slowly and tend to become stable. The adsorption capacities of methyl orange and Congo red can reach $223.66 \mathrm{mg} \mathrm{g}^{-1}$ and $347.19 \mathrm{mg} \mathrm{g}^{-1}$, respectively. Practical experiments for filtration/adsorption of dyes were carried out and are shown in the insets in Fig. 6. We crammed the GO/mPmPD/PVA aerogels into the bottoms of the funnels and added solutions containing the dyes into the funnels. The colors of the solutions disappeared and became clean water after flowing through the GO/mPmPD/PVA aerogel, which suggests that the dyes were adsorbed by the aerogel. In summary, GO/mPmPD/PVA aerogel is not only able to easily recover $\mathrm{Ag}(\mathrm{I})$, but also able to remove dyes.

\section{Conclusions}

In this study, we synthesized GO/mPmPD/PVA, a supramolecular-structure aerogel, through in situ polymerization and chemical crosslinking. Inspired by the crosslinking reaction between boric acid and PVA, 3-aminophenylboronic acid was introduced into the system for chemical crosslinking. A typical $B$ 1s peak appeared in the wide XPS spectrum of the GO/ $\mathrm{mPmPD} / \mathrm{PVA}$ aerogel suggesting that boric acid groups existed in the aerogel. The GO/mPmPD/PVA aerogel showed excellent hydrophilicity and great mechanical properties, which are of benefit for practical applications. The GO/mPmPD/PVA aerogel can adsorb $\mathrm{Ag}(\mathrm{I})$ with high efficiency, and the maximum $\operatorname{Ag}(\mathrm{I})$ adsorption capacity is $917.41 \mathrm{mg} \mathrm{g}^{-1}$. The $\mathrm{Ag}(\mathrm{I})$ adsorption isotherm fits better with the Langmuir isotherm model and the kinetics agree with the pseudo-second-order model. Chelation between $\mathrm{Ag}(\mathrm{I})$ and imine units and redox reactions between $\mathrm{Ag}(\mathrm{I})$ and benzenoid amine units are the main methods of $\operatorname{Ag}(\mathrm{I})$ adsorption. Furthermore, the GO/mPmPD/PVA aerogel also shows excellent adsorption ability for methyl orange and Congo red dyes. Practical experiments for the filtration/adsorption of 
dyes showed that solutions containing dyes become colorless after flowing through the GO/mPmPD/PVA aerogel. The GO/ $\mathrm{mPmPD} / \mathrm{PVA}$ supramolecular-structure aerogel shows enormous potential in applications for silver recovery and dye removal due to its effective adsorption capacity, low cost and facile separation with aqueous solution.

\section{Conflicts of interest}

There are no conflicts to declare.

\section{Acknowledgements}

This work was supported by the National Basic Research Program of China (2015CB932002), the National Natural Science Foundation of China (No. 21605144, 21876175, 21335006, 21275145, 21277145, 21375131, and 21475135), and the Key Research and Development Program of Anhui Province (1804a07020103).

\section{Notes and references}

1 X. Lu, Q. Yin, Z. Xin and Z. Zhang, Chem. Eng. Sci., 2010, 65, 6471-6477.

2 T. W. Purcell and J. J. Peters, Environ. Toxicol. Chem., 1998, 17, 539-546.

3 H. Huo, H. Su and T. Tan, Chem. Eng. J., 2009, 150, 139-144. 4 P. Leisner, C. Zanella, I. Belov, C. Edstrom, G. Sandulache and W. E. G. Hansal, Trans. Inst. Met. Finish., 2017, 95, 25-30.

5 L. Chai, T. Wang, L. Zhang, H. Wang, W. Yang, S. Dai, Y. Meng and X. Li, Carbon, 2015, 81, 748-757.

6 L. Li, C. Jia, F. Wang, H. Fan, W. Jiao and Z. Shao, J. Mater. Chem. C, 2018, 6, 2360-2369.

7 T. S. Anirudhan and M. Ramachandran, Process Saf. Environ. Prot., 2015, 95, 215-225.

8 W. Wang, G. Tian, Z. Zhang and A. Wang, Chem. Eng. J., 2015, 265, 228-238.

9 Y. Sun, Z.-Y. Wu, X. Wang, C. Ding, W. Cheng, S.-H. Yu and X. Wang, Environ. Sci. Technol., 2016, 50, 4459-4467.

10 M. Bhaumik, S. Agarwal, V. K. Gupta and A. Maity, J. Colloid Interface Sci., 2016, 470, 257-267.

11 H. N. M. E. Mahmud, A. K. O. Huq and R. B. Yahya, RSC Adv., 2016, 6, 14778-14791.

12 W. Yu, L. Zhang, H. Wang and L. Chai, J. Hazard. Mater., 2013, 260, 789-795.
13 T. Wang, L. Zhang, C. Li, W. Yang, T. Song, C. Tang, Y. Meng, S. Dai, H. Wang, L. Chai and J. Luo, Environ. Sci. Technol., 2015, 49, 5654-5662.

14 M. R. Huang, Q. Y. Peng and X. G. Li, Chem.-Eur. J., 2006, 12, 4341-4350.

15 X.-G. Li, X.-L. Ma, J. Sun and M.-R. Huang, Langmuir, 2009, 25, 1675-1684.

16 R. Tang, Q. Li, L. Ding, H. Cui and J. Zhai, Environ. Technol., 2012, 33, 341-348.

17 D. Chen, H. Feng and J. Li, Chem. Rev., 2012, 112, 6027-6053.

18 Y. Zhu, S. Murali, W. Cai, X. Li, J. W. Suk, J. R. Potts and R. S. Ruoff, Adv. Mater., 2010, 22, 3906-3924.

19 A. Iwase, Y. H. Ng, Y. Ishiguro, A. Kudo and R. Amal, J. Am. Chem. Soc., 2011, 133, 11054-11057.

20 W. Chen, S. Li, C. Chen and L. Yan, Adv. Mater., 2011, 23, 5679-5683.

21 Y. Qu, C. Lu, Y. Su, D. Cui, Y. He, C. Zhang, M. Cai, F. Zhang, X. Feng and X. Zhuang, Carbon, 2018, 127, 77-84.

22 K. Li, D. Guo, F. Lin, Y. Wei, W. Liu and Y. Kong, Electrochim. Acta, 2015, 166, 47-53.

23 K. Z. Setshedi, M. Bhaumik, M. S. Onyango and A. Maity, Chem. Eng. J., 2015, 262, 921-931.

24 J. Wu, Q. e. Zhang, J. Wang, X. Huang and H. Bai, Energy Environ. Sci., 2018, 11, 1280-1286.

25 K. Zhang, Y. Shi, S. Li, C. Wang, B. Yan, H. Xu, J. Wang, J. Guo and Y. Du, ChemElectroChem, 2017, 4, 1937-1943.

26 I. Saini, A. Sharma, R. Dhiman, S. Aggarwal, S. Ram and P. K. Sharma, J. Alloys Compd., 2017, 714, 172-180.

27 Y.-F. Huang, P.-F. Wu, M.-Q. Zhang, W.-H. Ruan and E. P. Giannelis, Electrochim. Acta, 2014, 132, 103-111.

28 H. H. Wang, T. W. Shyr and M. S. Hu, J. Appl. Polym. Sci., 1999, 74, 3046-3052.

29 D. C. Marcano, D. V. Kosynkin, J. M. Berlin, A. Sinitskii, Z. Sun, A. S. Slesarev, L. B. Alemany, W. Lu and J. M. Tour, ACS Nano, 2018, 12, 2078.

30 W. Wang, K. Cai, X. Wu, X. Shao and X. Yang, J. Alloys Compd., 2017, 722, 532-543.

31 L. Zhang, L. Chai, J. Liu, H. Wang, W. Yu and P. Sang, Langmuir, 2011, 27, 13729-13738.

32 J.-Y. Wang, T.-C. Chou, L.-C. Chen and K.-C. Ho, Biosens. Bioelectron., 2015, 63, 317-324.

33 Q. Kong, J. Wei, Y. Hu and C. Wei, J. Hazard. Mater., 2019, 363, 161-169.

34 X. Li, T. Liu, D. Wang, Q. Li, Z. Liu, N. Li, Y. Zhang, C. Xiao and X. Feng, ACS Appl. Mater. Interfaces, 2018, 10, 2167221680. 